\title{
Incremento de la ingesta de alcohol y drogas como estrategia de afrontamiento en trabajadores hospitalarios durante el brote de COVID-19: Estudio transversal
}

\author{
Self-reported increase in alcohol and drugs intake \\ as a coping strategy in hospital workers during \\ COVID-19 outbreak: A cross-sectional study
}

\author{
Agustín Madoz-Gúrpide*,**,***, Mónica Leira-Sanmartín*, Ángela Ibáñez*,**,***,****, \\ EnRiQueta OChOA-MANGADO*,**,***. \\ * Departamento de Psiquiatría. Hospital Universitario Ramón y Cajal, Madrid, España. \\ ** Instituto Ramón y Cajal de Investigación Sanitaria, Madrid, España. \\ *** Departamento de Medicina y Especialidades Médicas. Universidad de Alcalá, Madrid, España. \\ **** Centro de Investigación Biomédica en Red de Salud Mental (CIBERSAM).
}

\section{Resumen}

Las situaciones de estrés psicológico, como la actual pandemia COVID-19, pueden implicar un aumento del consumo de alcohol y otras drogas de abuso como estrategia inadecuada de afrontamiento en profesionales sanitarios. Esta investigación tiene como objetivo estudiar el incremento de la ingesta de alcohol y drogas de abuso en los trabajadores hospitalarios. Persigue también, mediante un análisis de regresión logística, definir qué perfil de trabajador es el más vulnerable a este comportamiento. Para ello se realizó un estudio transversal en un hospital terciario en Madrid, España, durante la primera oleada de COVID-19. Participaron en el estudio un total de 657 trabajadores del hospital, 536 de ellos sanitarios y 121 no sanitarios. La recogida de datos se realizó a través de una encuesta en línea que incluía preguntas sobre hábitos básicos de salud, condiciones del entorno laboral, datos sociodemográficos, así como la versión de 12 ítems del Cuestionario de Salud General. El 17,1\% declaró haber aumentado su consumo de alcohol y/o drogas de abuso durante el período analizado. Se asoció a una mayor probabilidad de dicho incremento: sexo masculino $(\mathrm{p}=$ ,044), vivir sin personas dependientes a cargo $(p=, 005)$, ser médico adjunto o residente $(p=, 010)$, haber trabajado en primera línea de COVID $(\mathrm{p}=, 058)$, presentar malos hábitos nutricionales $(\mathrm{p}=, 004)$ y realizar autoprescripción de fármacos psicotrópicos para controlar la ansiedad y el insomnio $(\mathrm{p}=, 003)$. Un porcentaje significativo de los trabajadores hospitalarios ha aumentado su consumo de alcohol y drogas de abuso durante la primera oleada de la pandemia COVID-19, existiendo un perfil de mayor riesgo para esta práctica.

Palabras clave: Infección por coronavirus 2019; personal de salud; estrés psicológico; factores de riesgo; factores protectores; abuso de alcohol; abuso de sustancias.

\section{Abstract}

Situations of psychological stress, such as the current COVID-19 pandemic, could lead to an increase in the consumption of alcohol and other drugs of abuse as an inadequate coping strategy in health workers. This study aimed to investigate the intake of alcohol and drugs of abuse in hospital workers during the first wave of COVID-19. A further focus was to define the worker profile most vulnerable to this behavior through a logistic regression analysis. A cross-sectional study in a tertiary hospital in Madrid, Spain, during the first wave of COVID-19 was designed. Information was collected from a sample (n $=657)$ of healthcare workers $(\mathrm{n}=536)$ and non-healthcare workers $(\mathrm{n}=121)$. An online survey (including questions about basic health habits, working environment conditions, sociodemographic data, and the 12-item version of the General Health Questionnaire as a measure of psychological well-being) was conducted. Increased consumption of alcohol and/or drugs of abuse during the analyzed period of the pandemic was reported by $17.1 \%$ of workers. The following variables were associated with a higher probability of increased consumption of alcohol and/or drugs of abuse: male gender $(p=.044)$, living alone or without dependents $(\mathrm{p}=.005)$, staff physician or resident $(\mathrm{p}=.010)$, having worked on the COVID frontline $(\mathrm{p}=.058)$, poor nutritional habits $(p=.004)$ and self-prescription of psychotropic drugs to manage anxiety and insomnia $(\mathrm{p}=.003)$. A significant percentage of hospital workers increased their consumption of alcohol and drugs of abuse during the first wave of the COVID-19 pandemic. A professional risk profile can be defined for this practice.

Key words: Coronavirus disease 2019 (COVID-19); health personnel; psychological stress; risk factors; protective factors; alcohol consumption; drug abuse. 
$\mathrm{P}$ uede suponerse que los profesionales de la salud llevan un estilo de vida más saludable que el resto de la población, pero tal vez esto no sea cierto: una investigación en la que participaron 7.288 médicos estadounidenses concluyó que el abuso/ dependencia del alcohol es un problema importante entre estos trabajadores (el 12,9\% y el 21,4\% de los médicos hombres/mujeres respectivamente cumplen los criterios de abuso o dependencia del alcohol) (Oreskovich et al., 2015). En Alemania, una encuesta en la que participaron estudiantes de Medicina y médicos mostró que el 24,8\% de los médicos varones y el $36,5 \%$ de los estudiantes de Medicina varones referían ingestas superiores a la dosis diaria recomendada por la Sociedad Alemana de Nutrición sobre la ingesta máxima de alcohol (>20 g/d). Entre las mujeres participantes, el 25,3\% de los médicos y el 30,4\% de los estudiantes de Medicina informaron de que habían ingerido más alcohol del que se les recomendaba (> 10 g/d) (Voigt et al., 2009). En una muestra brasileña de 510 médicos y enfermeras, estos profesionales mostraron una menor prevalencia de abstemios, tasas similares de uso indebido de alcohol y una mayor prevalencia de episodios de consumo excesivo de alcohol en comparación con la población general brasileña (Tobias et al., 2019). En España, una encuesta reciente entre los profesionales de la Atención Primaria mostró una mayor prevalencia del consumo de alcohol en este colectivo con respecto a la población general, con un porcentaje de consumo de riesgo según los criterios de la AUDIT-C del 32\% (Romero-Rodríguez et al., 2019a). El perfil de un bebedor de riesgo en esta muestra era el de un varón, médico, mayor de 56 años y con más años promedio de carrera profesional (Romero-Rodríguez et al., 2019b).

Se sabe poco sobre el comportamiento de salud y los estilos de vida que contribuyen al uso abusivo del alcohol y otras drogas entre los profesionales de la salud. Hay diferentes variables que se han relacionado con un mayor riesgo de uso indebido de alcohol y drogas entre los trabajadores sanitarios: rasgos de personalidad como la alexitimia (Pedersen, Sørensen, Bruun, Christensen y Vedsted, 2016), estilos de afrontamiento (Grotmol et al., 2010), factores personales y profesionales, y el hecho de verse afectado por el "síndrome de burnout" (Alexandrova-Karamanova et al., 2016). El uso indebido de drogas y alcohol también ha sido considerado frecuentemente como una estrategia disfuncional de automedicación ante la comorbilidad con los trastornos afectivos y de ansiedad en la población general (Robinson, Sareen, Cox y Bolton, 2009; Turner, Mota, Bolton y Sareen, 2018). Lo mismo podría decirse del consumo de alcohol como medida para controlar los síntomas del trastorno de estrés postraumático (Bensley et al., 2018).

La pandemia de COVID-19 ha establecido una serie de condiciones ambientales, sociales e intrapsíquicas que po- drían haber promovido el uso inadecuado del alcohol y otras drogas como recurso para controlar la angustia psicológica. Un editorial de The Lancet señaló el aumento de los marcadores directos e indirectos del consumo de alcohol en la población general durante la pandemia, tanto en personas sin patrones de consumo de riesgo previos como en individuos con trastornos de consumo de alcohol (Clay y Parker, 2020). Existen pruebas recientes que muestran una asociación significativa entre el estrés psicológico específico de COVID-19 y la conducta de consumo de alcohol, estando la relación mediada por el sexo y por las consecuencias socioeconómicas de la pandemia en la población general estadounidense (Rodriguez, Litt y Stewart, 2020; Wardell et al., 2020). Existe una creciente preocupación por un posible empeoramiento durante la pandemia de la carga global de enfermedad relacionada con los trastornos por consumo de alcohol, incluida la creciente incidencia de enfermedades hepáticas relacionadas con el alcohol (Da, Im y Schiano, 2020). La relación entre el uso indebido de sustancias y la pandemia de COVID-19 también se ha estudiado en otros países y entornos (Neill et al., 2020). Informes anteriores sobre la relación entre la exposición de los trabajadores de la salud a brotes pandémicos y el riesgo de desarrollar un trastorno de consumo de alcohol indican que, el hecho de haber estado en cuarentena y de trabajar en lugares donde la exposición a pacientes de SARS era común, se asociaba con síntomas posteriores de abuso/dependencia del alcohol, incluso tres años después del brote de SARS (Wu et al., 2008).

El objetivo de este estudio es estimar si hubo un aumento en la ingesta de alcohol y drogas de abuso en trabajadores de hospitales durante la primera oleada de COVID-19. Además, se pretende definir qué perfil de trabajador es el más vulnerable a este comportamiento.

Desde mediados de marzo hasta finales de abril de 2020, España sufrió la primera oleada del brote de COVID-19, que afectó especialmente a la ciudad de Madrid. El hospital donde se realizó el estudio (Hospital Universitario Ramón y Cajal), un gran centro de atención terciaria, fue uno de los más golpeados por la pandemia, teniendo que afrontar una profunda reestructuración logística y asistencial para atender la demanda generada por el pico de hospitalizaciones por neumonía causada por el SARS-CoV-2.

\section{Método}

Se trata es un estudio transversal, para la realización del cual se diseñó un formulario para ser usado en una encuesta online entre todo el personal que trabajaba en el Hospital Universitario Ramón y Cajal durante la primera oleada de COVID-19. La encuesta fue distribuida en línea por correo institucional, y estuvo también accesible desde la intranet del hospital. Se animó a todos los trabajadores de diferentes categorías a participar, de manera anóni- 
ma, en un estudio voluntario y sin incentivo económico. El formulario se dividió en cuatro secciones, que agrupaban diferentes tipos de variables: datos sociodemográficos (sexo, edad, tipo de convivencia familiar) y estado de salud y profesional durante la pandemia (categoría profesional, experiencia, tipo de actividad, antecedentes personales de salud mental, infección por los síntomas del SARS-CoV-2, COVID-19); factores de estrés que los trabajadores relacionaron con el ambiente de trabajo y las actividades durante la pandemia; conductas de riesgo y protección fuera del lugar de trabajo durante la pandemia; y la escala del Cuestionario de Salud General (GHQ-12) (Goldberg y Hillier, 1979; Goldberg y Williams, 1988). Este cuestionario es una herramienta de detección de trastornos mentales comunes, así como una medida general de bienestar psicológico, que está validado para su uso en población española (Sánchez-López y Dresch, 2008). Entre las diferentes conductas de riesgo se interrogaba específicamente a los encuestados si habían incrementado el consumo de alcohol y/o drogas de abuso durante el periodo de la primera ola de COVID-19 (mediados de marzo a finales de abril). La pregunta “ ¿Ha incrementado durante la pandemia el consumo de alcohol o drogas de abuso?" cuestiona sobre el uso de dichas sustancias como una posible manera de afrontar el estrés generado por la situación de pandemia.

La encuesta se realizó entre el 15 de junio y el 25 de julio de 2020 y fue aprobada previamente por el Comité Ético de Investigación Clínica del hospital. Se requirió el consentimiento informado de todos los individuos antes de participar.

Inicialmente, se realizó un análisis crudo de los resultados. Para aumentar el poder del análisis, algunas variables fueron recodificadas y se agruparon siguiendo criterios que se consideraron clínicamente relevantes. Las variables continuas se describieron mediante la media y la desviación estándar (DE). Las variables categóricas, a través de la frecuencia absoluta y la relativa. Para la estadística inferencial se utilizó la prueba de la t de Student para las variables cuantitativas. La asociación entre las variables categóricas se hizo mediante la prueba de chi cuadrado o la prueba exacta de Fisher. Para estudiar la asociación entre el uso de alcohol y/o drogas de abuso y las variables de riesgo, se utilizó el modelo de regresión logística por pasos hacia atrás (Wald), ajustando por las variables que, en función de la revisión bibliográfica, los resultados brutos de nuestro estudio y la plausibilidad biológica, se interpretó que pudieran tener influencia en el uso indebido de drogas y alcohol. Esta asociación se presenta como una razón de odds (OR). Se exploró la posible existencia de interacción y confusión. Todos los análisis se realizaron con el Paquete Estadístico para las Ciencias Sociales (SPSS), versión 19.

\section{Resultados}

657 personas respondieron a la encuesta (sobre un total de 6.119 profesionales sanitarios y no sanitarios, entre plantilla estable y refuerzos contratados para atender la primera ola COVID). De ellas, el 79,1\% eran mujeres, con una edad media estimada de 41,06 años (DE $=11,63)$. El $33,5 \%$ vivía con personas dependientes a su cargo, mientras que el 51,4\% vivía solo o en pareja. El 81,6\% de los encuestados eran trabajadores sanitarios. El 28,9\% de los que respondieron a la encuesta eran enfermeros y el 17,0\% auxiliares de enfermería. El 13,5\% eran médicos residentes y el 19,8\% médicos de plantilla. La distribución por categoría profesional en la muestra es similar a la de población total de trabajadores del hospital durante la pandemia. 15,3 fueron los años promedio de experiencia profesional (DE $=10,9)$. El 54,3\% trabajaba directamente en el cuidado de los pacientes con COVID-19 (los llamados trabajadores de “primera línea”). De los encuestados, el 17,1\% declaró haber aumentado el consumo de alcohol y/o drogas de abuso durante el período analizado de la pandemia.

Hubo diferencias significativas en ciertas variables entre los que aumentaron el consumo de alcohol o utilizaron drogas de abuso, y los que no lo hicieron. Cabe destacar que los que aumentaron el consumo de alcohol/drogas durante el brote de COVID eran significativamente más jóvenes $(p=, 005)$, y con una mayor puntuación total de GHQ-12 ( $\mathrm{p}=, 031)$ que se interpreta como un mayor nivel de estrés psicológico o impacto emocional (Tabla 1). Las diferencias en el tipo de convivencia también fueron

Tabla 1. Incremento de consumo de alcohol/drogas de abuso y variables sociodemográficas.

\begin{tabular}{|c|c|c|c|c|c|}
\hline & Incremento de consumo de alcohol/drogas de abuso & $\mathbf{n}$ & Media & DE & Sig. \\
\hline \multirow{2}{*}{ Edad media estimada } & No & 542 & 41,65 & 11,83 & \multirow{2}{*}{, 005} \\
\hline & Sí & 112 & 38,25 & 10,85 & \\
\hline \multirow{2}{*}{$\begin{array}{l}\text { Media de años de experiencia } \\
\text { profesional }\end{array}$} & No & 454 & 15,67 & 11,00 & \multirow{2}{*}{,071 } \\
\hline & Sí & 107 & 13,57 & 10,07 & \\
\hline \multirow{2}{*}{ Puntuación GHQ-12 } & No & 534 & 16,62 & 5,40 & \multirow{2}{*}{,031 } \\
\hline & Sí & 110 & 17,85 & 5,79 & \\
\hline
\end{tabular}

DE: desviación estándar.

GHQ-12: Cuestionario de Salud General de Goldberg, versión 12 ítems. 
significativas (mayor porcentaje de convivencia sin dependientes a cargo entre los que aumentaron el consumo, $p$ $=, 001)$. Los que incrementaron el consumo de alcohol o drogas de abuso eran más frecuentemente profesionales sanitarios $(p=, 022)$, habían trabajado en primera línea contra COVID-19 ( $\mathrm{p}=, 009)$, y tenían una mayor representación de médicos de plantilla y residentes $(\mathrm{p}=, 001)$ ( Tabla 2). También se observan diferencias significativas entre variables consideradas como factores de protección o riesgo: quienes han incrementado el consumo de alcohol/ drogas de abuso presentan peores hábitos nutricionales ( $\mathrm{p}$ $=, 001)$, y también usan con más frecuencia psicótropos autoprescritos $(p=, 000)$. No se apreciaron diferencias estadísticamente significativas en cuanto a la presencia de antecedentes personales de enfermedad mental $(\mathrm{p}=, 575)$. (Tablas 2 y 3 ).

En el modelo de regresión logística (Tabla 4) destacan significativamente los siguientes hallazgos: ser varón se re-

Tabla 2. Análisis de comparación de variables independientes vs Incremento de consumo de alcohol/drogas de abuso.

\begin{tabular}{|c|c|c|c|c|c|c|c|}
\hline & & \multicolumn{4}{|c|}{ Incremento de consumo de alcohol/drogas de abuso } & \multirow{3}{*}{ Chi cuadrado } & \multirow{3}{*}{ Sig. } \\
\hline & & \multicolumn{2}{|c|}{ No } & \multicolumn{2}{|c|}{ Sí } & & \\
\hline & & $\mathbf{n}$ & $\%$ & $\mathbf{n}$ & $\%$ & & \\
\hline \multirow{2}{*}{ Sexo } & Hombre & 106 & $19,6 \%$ & 30 & $27,0 \%$ & \multirow{2}{*}{3,118} & \multirow{2}{*}{,095 } \\
\hline & Mujer & 436 & $80,4 \%$ & 81 & $73,0 \%$ & & \\
\hline \multirow{2}{*}{ Tipo de convivencia } & Sin personas a cargo & 261 & $48,2 \%$ & 74 & $66,1 \%$ & \multirow{2}{*}{11,804} & \multirow{2}{*}{, 001} \\
\hline & Con personas a cargo & 280 & $51,8 \%$ & 38 & $33,9 \%$ & & \\
\hline \multirow{2}{*}{ Profesional sanitario } & Sí & 433 & $79,9 \%$ & 100 & $89,3 \%$ & \multirow{2}{*}{5,435} & \multirow{2}{*}{,022 } \\
\hline & No & 109 & $20,1 \%$ & 12 & $10,7 \%$ & & \\
\hline \multirow{2}{*}{ Contacto estrecho con COVID-19 } & Primera línea & 273 & $50,4 \%$ & 72 & $64,3 \%$ & \multirow{2}{*}{7,213} & \multirow{2}{*}{, 009} \\
\hline & Segunda línea & 269 & $49,6 \%$ & 40 & $35,7 \%$ & & \\
\hline \multirow{2}{*}{ Médico de plantilla y Residentes } & Sí & 166 & $30,6 \%$ & 53 & $47,3 \%$ & \multirow{2}{*}{11,614} & \multirow{2}{*}{, 001} \\
\hline & No & 376 & $69,4 \%$ & 59 & $52,7 \%$ & & \\
\hline \multirow{2}{*}{ Screening positivo en GHQ-12 } & Sí & 449 & $84,1 \%$ & 93 & $84,5 \%$ & \multirow{2}{*}{0,015} & \multirow{2}{*}{1,000} \\
\hline & No & 85 & $15,9 \%$ & 17 & $15,5 \%$ & & \\
\hline
\end{tabular}

GHQ-12: Cuestionario de Salud General de Goldberg, versión 12 ítems.

Tabla 3. Análisis de comparación de factores de riesgo y de protección vs Incremento de consumo de alcohol/drogas de abuso.

\begin{tabular}{|c|c|c|c|c|c|c|c|}
\hline & & \multicolumn{4}{|c|}{ Incremento de consumo de alcohol/drogas de abuso } & \multirow{3}{*}{ Chi cuadrado } & \multirow{3}{*}{ Sig. } \\
\hline & & \multicolumn{2}{|c|}{ No } & \multicolumn{2}{|c|}{ Sí } & & \\
\hline & & $\mathbf{n}$ & $\%$ & $\mathbf{n}$ & $\%$ & & \\
\hline \multirow{2}{*}{ Presencia de sintomatología COVID-19 } & No & 326 & $60,3 \%$ & 60 & $53,6 \%$ & \multirow{2}{*}{1,717} & \multirow{2}{*}{, 206} \\
\hline & Sí & 215 & $39,7 \%$ & 52 & $46,4 \%$ & & \\
\hline \multirow{2}{*}{ Diagnóstico clínico de infección COVID-19 } & No & 344 & $63,9 \%$ & 76 & $69,1 \%$ & \multirow{2}{*}{1,062} & \multirow{2}{*}{, 326} \\
\hline & Sí & 194 & $36,1 \%$ & 34 & $30,9 \%$ & & \\
\hline \multirow{2}{*}{ Pérdida de allegado } & No & 420 & $78,1 \%$ & 93 & $83,8 \%$ & \multirow{2}{*}{1,816} & \multirow{2}{*}{,201 } \\
\hline & Sí & 118 & $21,9 \%$ & 18 & $16,2 \%$ & & \\
\hline \multirow{2}{*}{ Actividad física regular } & No & 328 & $60,7 \%$ & 75 & $67,0 \%$ & \multirow{2}{*}{1,522} & \multirow{2}{*}{, 240} \\
\hline & Sí & 212 & $39,3 \%$ & 37 & $33,0 \%$ & & \\
\hline \multirow{2}{*}{ Práctica de relajación/meditación/mindfulness } & No & 341 & $63,0 \%$ & 69 & $61,6 \%$ & \multirow{2}{*}{0,081} & \multirow{2}{*}{, 830} \\
\hline & Sí & 200 & $37,0 \%$ & 43 & $38,4 \%$ & & \\
\hline \multirow{2}{*}{ Hábitos higiénicos de sueño } & Inadecuado & 277 & $51,1 \%$ & 62 & $55,4 \%$ & \multirow{2}{*}{0,672} & \multirow{2}{*}{,467 } \\
\hline & Adecuado & 265 & $48,9 \%$ & 50 & $44,6 \%$ & & \\
\hline \multirow{2}{*}{ Hábitos nutricionales } & Inadecuado & 206 & $38,1 \%$ & 62 & $55,9 \%$ & \multirow{2}{*}{12,025} & \multirow{2}{*}{, 001} \\
\hline & Adecuado & 335 & $61,9 \%$ & 49 & $44,1 \%$ & & \\
\hline \multirow{2}{*}{ Actividades de ocio } & Inadecuado & 368 & $68,3 \%$ & 82 & $73,2 \%$ & \multirow{2}{*}{1,060} & \multirow{2}{*}{,369 } \\
\hline & Adecuado & 171 & $31,7 \%$ & 30 & $26,8 \%$ & & \\
\hline \multirow{2}{*}{ Interacción social } & Inadecuado & 232 & $42,9 \%$ & 47 & $42,0 \%$ & \multirow{2}{*}{0,032} & \multirow{2}{*}{,917 } \\
\hline & Adecuado & 309 & $57,1 \%$ & 65 & $58,0 \%$ & & \\
\hline Exposición a información en medios y redes sociales & Inadecuado & 198 & $37,0 \%$ & 51 & $45,5 \%$ & 2844 & 109 \\
\hline 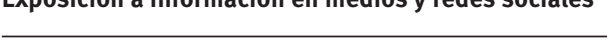 & Adecuado & 337 & $63,0 \%$ & 61 & $54,5 \%$ & 2,044 &, 108 \\
\hline Antecedentes nersonales de enfermedad mental & No & 453 & $84,2 \%$ & 92 & $82,1 \%$ & 0.290 & 575 \\
\hline 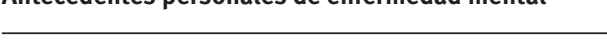 & Sí & 85 & $15,8 \%$ & 20 & $17,9 \%$ & $0,2,0$ & שו \\
\hline Autoprescripción de psicotropos & No & 409 & $75,6 \%$ & 64 & $57,1 \%$ & 15.833 & 000 \\
\hline Autoprescripcton de psicotropos & Sí & 132 & $24,4 \%$ & 48 & $42,9 \%$ & 15,833 &, 000 \\
\hline
\end{tabular}


Tabla 4. Modelo de regresión.

\begin{tabular}{lcccccccc}
\hline & & & & & & & \multicolumn{2}{c}{ IC95\% EXP(B) } \\
\cline { 6 - 9 } & B & E.T. & Wald & df & Sig & Exp (B) & Inferior & Superior \\
\hline Sexo &, 544 &, 270 & 4,043 & 1 &, 044 & 1,723 & 1,014 & 2,927 \\
Tipo de convivencia &, 659 &, 235 & 7,848 & 1 &, 005 & 1,934 & 1,219 & 3,067 \\
Autoprescripción de psicotropos &, 733 &, 243 & 9,122 & 1 &, 003 & 2,081 & 1,293 & 3,348 \\
Hábitos nutricionales &, 702 &, 242 & 8,433 & 1 &, 004 & 2,018 & 1,256 & 3,241 \\
Médico de plantilla y Residentes &, 614 &, 240 & 6,567 & 1 &, 010 & 1,847 & 1,155 & 2,954 \\
Contacto estrecho con COVID-19 & $, 4,50$ &, 237 & 3,608 & 1 &, 058 & 1,568 &, 986 & 2,493 \\
Actividades de ocio & $-9,107$ & 1,364 & 44,604 & 1 &, 000 &, 000 & & \\
\hline
\end{tabular}

Variables introducidas en el modelo: Sexo, edad media estimada, años de experiencia, profesional sanitario, presencia de sintomatología COVID-19, pérdida de allegado, antecedentes personales de enfermedad mental, tipo de convivencia, contacto estrecho con COVID-19, autoprescripción de psicótropos, hábitos nutricionales, hábitos higiénicos de sueño, actividades de ocio, interacción social, actividad física regular, médico de plantilla y residentes, puntuación GHQ-12.

lacionaba con un incremento del riesgo de aumentar la ingesta de alcohol/drogas ilícitas en 1,723 (IC 95\%: 1,014 a 2,927), si el resto de variables permanecían constantes. También el hecho de vivir solo o con una pareja (no tener personas dependientes a cargo) incrementaba el riesgo de aumentar el consumo de alcohol/drogas ilícitas en 1,934 (IC 95\%: 1,014 a 2,927) frente al riesgo de quienes vivían con personas a su cargo. Si todos los valores de las otras variables del modelo permanecen constantes y se produce un uso de psicotrópicos autoprescritos, el riesgo de incrementar el consumo de alcohol/drogas de abuso aumenta 2,081 (95\% CI: 1,293 a 3,348) veces más que el riesgo si no se autoprescribe el uso de psicotropos. Ser un médico de plantilla o un interno residente también fue un factor a considerar, aumentando en 1,847 (95\% CI: 1,155 a 2,954) el riesgo de incrementar el uso de alcohol/drogas frente al del resto de categorías profesionales. Asimismo, trabajar en la primera línea de COVID-19 aumentó el riesgo de incrementar el uso de alcohol/drogas ilícitas en 1,658 (95\% CI: 0,986 a 2,493). Finalmente, quienes tenían malos hábitos nutricionales tenían un riesgo de incrementar el consumo de alcohol/drogas ilícitas en 2,018 (95\% CI: 1,256 a 3,241) más que quienes mantenían buenos hábitos.

\section{Discusión}

Un porcentaje elevado de la muestra reconoció un incremento de la ingesta de alcohol y/o drogas de abuso durante la primera ola de COVID-19. Quienes han incrementado dicha ingesta, respecto a quienes no lo han hecho, son más jóvenes, forman con más frecuencia parte del grupo de los profesionales sanitarios, con mayor representación de médicos de plantilla y residentes, han trabajado más frecuentemente en primera línea de atención a pacientes COVID-19, y viven en mayor porcentaje solos o sin personas a su cargo. Tienen además peores hábitos alimentarios y refieren un mayor estrés medido a través de la escala GHQ-12. También con más frecuencia se han autoprescrito fármacos psicotrópicos para gestionar la ansiedad y el insomnio. Quienes habían incrementado el consumo de sustancias durante la pandemia no tenían una mayor frecuencia de antecedentes personales de seguimiento en Salud Mental.

Hasta la fecha, hay escasos informes sobre el uso indebido de alcohol y drogas en los trabajadores de la salud durante el brote de COVID-19 (Conroy et al., 2021; Gomes et al., 2020; Silczuk, 2020). Investigaciones previas han señalado un incremento significativo del consumo de alcohol entre los médicos que estaban confinados (Silczuk, 2020), pero también entre los trabajadores sanitarios sin importar si trabajaban de forma presencial o desde sus casas (Conroy et al., 2021). En este último estudio, el $8 \%$ de la muestra era consumidor de cannabis antes del confinamiento, refiriendo asimismo un aumento en la cantidad consumida de esta sustancia durante la pandemia COVID-19 (Conroy et al., 2021). El incremento del consumo de alcohol en otros estudios fue identificado como una manifestación concreta del empeoramiento global de los hábitos de salud entre los médicos, estando así relacionado con el aumento de peso y la disminución de la actividad física y sexual (Gomes et al., 2020).

En nuestra muestra, los profesionales más jóvenes que vivían sin personas dependientes a su cargo estaban más predispuestos a desarrollar un incremento del uso de alcohol o del consumo de drogas de abuso. Los médicos y los varones estaban más representados en este grupo, y la puntuación total en el GHQ-12 tendía a ser más alta entre ellos respecto al promedio de la muestra.

En algunos informes anteriores se ha señalado que los estudiantes de medicina/médicos residentes varones consumen alcohol con mayor regularidad en comparación con otros profesionales sanitarios (Lamberti et al., 2017). En nuestro estudio, se encontró una puntuación total de GHQ12 más alta $(\mathrm{p}=, 041)$ en aquellos que incrementaron el uso de alcohol y/o drogas de abuso. Trabajar en primera línea durante la pandemia COVID-19 se asoció significativamente con el aumento del uso de alcohol y drogas ilícitas, también. Esto nos lleva a concluir que el uso de alcohol o drogas de abuso puede representar una estrategia de afrontamiento disfuncional para manejar los síntomas de ansiedad/insom- 
nio, en un grupo de trabajadores de la salud que tienen menos experiencia y pueden ser más vulnerables al estrés, además de carecer de las herramientas de afrontamiento necesarias. En la población general, la automedicación para la ansiedad mediante el consumo de alcohol y drogas también se ha notificado con mayor frecuencia en personas más jóvenes y en las que están divorciadas o nunca se han casado (Robinson et al., 2009). Los que no se automedican son con mayor frecuencia mujeres (Robinson et al., 2009). En el contexto de la pandemia COVID-19, las investigaciones basadas en la población general han encontrado que los padres con al menos un hijo menor de 18 años tenían más riesgo de aumentar el consumo de alcohol (Wardell et al., 2020), lo que es contrario a nuestros hallazgos sobre la asociación de vivir sin personas dependientes con un mayor riesgo de abuso de alcohol/drogas.

Nuestro estudio incluye algunas limitaciones que merece la pena considerar. Es difícil, por un lado, estimar hasta qué punto quienes responden a la encuesta son una muestra representativa de los trabajadores del hospital, aunque la representación por categoría profesional de la muestra si es similar a la de la población de estudio. Por otra parte, la variable que mide el incremento del consumo de sustancias presupone que esta es una estrategia de afrontamiento del estrés que generó la situación de pandemia. Supone también una limitación la ausencia de documentación de patrones previos de uso de alcohol y/o drogas en los sujetos del estudio, y la ausencia del uso de herramientas estandarizadas para estimar y cuantificar el consumo, utilizando exclusivamente el autoinforme genérico de los individuos. Esto podría a su vez estar condicionando la falta de correlación entre dicho consumo y los antecedentes previos de enfermedad mental. Asimismo, es probable que exista un sesgo de información al solicitar a los encuestados que recordaran eventos y datos acaecidos unos meses antes, y además en una situación de estrés y gran impacto emocional. Igualmente es más probable que respondieran al cuestionario aquellos que más sufrieron el impacto psicológico de la primera oleada (sesgo de selección). Por otro lado, el tipo de estudio realizado no permite establecer asociaciones causales sino relaciones entre variables.

La relevancia de estos hallazgos sobre los patrones de uso de drogas y alcohol en los trabajadores de los hospitales durante el brote de Covid-19, indica la necesidad de monitorizar la evolución de los sujetos que aumentaron su consumo de alcohol o tomaron drogas ilícitas para hacer frente a la ansiedad, y a adoptar intervenciones específicas de detección y terapéuticas que disminuya el impacto del uso indebido de sustancias en los trabajadores de los hospitales.

\section{Conclusiones}

Un porcentaje significativo de los trabajadores del hospital que lucharon contra el COVID-19 durante la prime- ra oleada en el Hospital Universitario Ramón y Cajal, en Madrid, aumentó su consumo de alcohol y/o drogas de abuso. Dado el perfil que presenta este grupo de profesionales (mayor exposición al estrés y ausencia de otras estrategias de afrontamiento), se sugiere que el uso de alcohol y drogas de abuso hayan supuesto una estrategia fallida para afrontar el estrés y la ansiedad producidos por la situación de pandemia, en individuos que tienen menos experiencia y pueden ser más vulnerables a los mismos. Es importante implementar tanto las pruebas de detección como las intervenciones terapéuticas que favorezcan un enfoque adecuado de este importante problema de salud en los trabajadores de los hospitales.

\section{Conflicto de intereses}

Los autores declaran que no hay conflicto de intereses en este trabajo.

\section{Referencias}

Alexandrova-Karamanova, A., Todorova, I., Montgomery, A., Panagopoulou, E., Costa, P., Baban, A.,... Mijakoski, D. (2016). Burnout and health behaviors in health professionals from seven European countries. International Archives of Occupational and Environmental Health, 89, 1059-1075. doi:10.1007/s00420-016-1143-5.

Bensley, K. M., Seelig, A. D., Armenta, R. F., Rivera, A. C., Peterson, A. V., Jacobson, I. G.,... Williams, E. C. (2018). Posttraumatic stress disorder symptom association with subsequent risky and problem drinking initiation. Journal of Addiction Medicine, 12, 353-362. doi:10.1097/ ADM.0000000000000420.

Clay, J. M. y Parker, M. O. (2020). Alcohol use and misuse during the COVID-19 pandemic: A potential public health crisis? Lancet Public Health, 5. doi:10.1016/S24682667(20)30088-8.

Conroy, D. A., Hadler, N. L., Cho, E., Moreira, A., MacKenzie, C., Swanson, L. M.,... Goldstein, C. A. (2021). The effects of COVID-19 stay-at-home order on sleep, health, and working patterns: A survey study of US health care workers. Journal of Clinical Sleep Medicine, 17, 185-191. doi:10.5664/jcsm.8808.

Da, B. L., Im, G. Y. y Schiano, T. D. (2020). Coronavirus disease 2019 hangover: A rising tide of alcohol use disorder and alcohol-associated liver disease. Hepatology, 72, 1102-1108. doi:10.1002/hep.31307.

Goldberg, D. P. y Hillier, V. F. (1979). A scaled version of the general health questionnaire. Psychological Medicine, 9, 139-145. doi:10.1017/S0033291700021644.

Goldberg, D. P. y Williams, P. (1988). A user's guide to the general health questionnaire. Berks: Windsor: NFER-Nelson.

Gomes, C. M., Favorito, L. A., Henriques, J. V. T., Canalini, A. F., Anzolch, K. M. J., de Carvalho Fernandes, R.,... 
de Bessa, J. (2020). Impact of COVID-19 on clinical practice, income, health and lifestyle behavior of Brazilian urologists. International Braz J Urol, 46, 1042-1056. doi:10.1590/S1677-5538.IBJU.2020.99.15.

Grotmol, K. S., Vaglum, P., Ekeberg, Ø., Gude, T., Aasland, O. G. y Tyssen, R. (2010). Alcohol expectancy and hazardous drinking: A 6-year longitudinal and nationwide study of medical doctors. European Addiction Research, 16, 17-22. doi:10.1159/000253860.

Lamberti, M., Napolitano, F., Napolitano, P., Arnese, A., Crispino, V., Panariello, G. y di Giuseppe, G. (2017). Prevalence of alcohol use disorders among under- and post-graduate healthcare students in Italy. PLoS ONE, 12. doi:10.1371/journal.pone.0175719.

Neill, E., Meyer, D., Toh, W. L., van Rheenen, T. E., Phillipou, A., Tan, E. J. y Rossell, S. L. (2020). Alcohol use in Australia during the early days of the COVID-19 pandemic: Initial results from the COLLATE project. Psychiatry and Clinical Neurosciences, 74, 542-549. doi:10.1111/ pcn.13099.

Oreskovich, M. R., Shanafelt, T., Dyrbye, L. N., Tan, L., Sotile, W., Satele, D.,... Boone, S. (2015). The prevalence of substance use disorders in American physicians. American Journal on Addictions, 24, 30-38. doi:10.1111/ ajad.12173.

Pedersen, A. F., Sørensen, J. K., Bruun, N. H., Christensen, B. y Vedsted, P. (2016). Risky alcohol use in Danish physicians: Associated with alexithymia and burnout? Drug and Alcohol Dependence, 160, 119-126. doi:10.1016/j.drugalcdep.2015.12.038.

Robinson, J., Sareen, J., Cox, B. J. y Bolton, J. (2009). Self-medication of anxiety disorders with alcohol and drugs: Results from a nationally representative sample. Journal of Anxiety Disorders, 23, 38-45. doi:10.1016/j.janxdis.2008.03.013.

Rodriguez, L. M., Litt, D. M. y Stewart, S. H. (2020). Drinking to cope with the pandemic: The unique associations of COVID-19-related perceived threat and psychological distress to drinking behaviors in American men and women. Addictive Behaviors, 110. doi:10.1016/j.addbeh.2020.106532.

Romero-Rodríguez, E., Pérula De Torres, L. Á., Fernández García, J. Á., Parras Rejano, J. M., Roldán Villalobos, A. y Camarelles Guillén, F. (2019a). Alcohol consumption in Spanish primary health care providers: A national, cross-sectional study. BMJ Open, 9. doi:10.1136/bmjopen-2018-024211.

Romero-Rodríguez, E., Pérula De Torres, L. Á., Parras Rejano, J. M., Leiva-Cepas, F., Camarelles Guillem, F., Fernández Márquez, R.,... Pérula De Torres, C. (2019b). Prevalence of hazardous alcohol use among Spanish primary care providers. BMC Family Practice, 20. doi:10.1186/s12875-019-0999-3.
Sánchez-López, M. D. P. y Dresch, V. (2008). The 12-item general health questionnaire (GHQ-12): Reliability, external validity and factor structure in the Spanish population. Psicothema, 20, 839-843.

Silczuk, A. (2020). Threatening increase in alcohol consumption in physicians quarantined due to coronavirus outbreak in Poland: The ALCOVID survey. Journal of Public Health, 42, 461-465. doi:10.1093/pubmed/fdaa110.

Tobias, J. S. P., da Silva, D. L. F., Ferreira, P. A. M., da Silva, A. A. M., Ribeiro, R. S. y Ferreira, A. S. P. (2019). Alcohol use and associated factors among physicians and nurses in northeast Brazil. Alcohol, 75, 105-112. doi:10.1016/j. alcohol.2018.07.002.

Turner, S., Mota, N., Bolton, J. y Sareen, J. (2018). Self-medication with alcohol or drugs for mood and anxiety disorders: A narrative review of the epidemiological literature. Depression and Anxiety, 35, 851-860. doi:10.1002/ da. 22771.

Voigt, K., Twork, S., Mittag, D., Göbel, A., Voigt, R., Klewer, J.,... Bergmann, A. (2009). Consumption of alcohol, cigarettes and illegal substances among physicians and medical students in Brandenburg and Saxony (Germany). BMC Health Services Research, 9, 219. doi:10.1186/1472-6963-9-219.

Wardell, J. D., Kempe, T., Rapinda, K. K., Single, A., Bilevicius, E., Frohlich, J. R.,... Keough, M. T. (2020). Drinking to cope during COVID-19 pandemic: The role of external and internal factors in coping motive pathways to alcohol use, solitary drinking, and alcohol problems. Alcoholism, Clinical and Experimental Research, 44, 2073-2083. doi:10.1111/acer.14425.

Wu, P., Liu, X., Fang, Y., Fan, B., Fuller, C. J., Guan, Z., .. Litvak, I. J. (2008). Alcohol abuse/dependence symptoms among hospital employees exposed to a SARS outbreak. Alcohol and Alcoholism, 43, 706-712. doi:10.1093/alcalc/ agn073. 
